\title{
Investigação da carga parasitária em aves destinadas ao consumo humano, em feira do Município de Porto Velho-RO, Brasil
}

Investigation of the parasite load in birds destined for human consumption, at a fair in the City of Porto Velho-RO, Brazil

Investigación de la carga parasitaria en aves destinadas al consumo humano, en una feria en la Ciudad de Porto Velho-RO, Brasil

\section{Resumo}

Este estudo teve como objetivo estabelecer a prevalência de parasitoses em galinhas domesticadas, criadas em regime extensivo no município de Porto Velho, Rondônia, Brasil. Foram analisadas 40 amostras fecais de aves em 4 (quatro) propriedades, sendo 10 em cada uma delas. As amostras foram então processadas pela técnica qualitativa de Willis \& Mollay (flutuação simples, 1921). Das 40 amostras de fezes analisadas, 32 foram positivas e 8 negativas e destas 32,9 apresentaram infecções mistas. Os ovos dos parasitos que foram encontrados nos exames são os seguintes: Heterakis $s p$, Capillaria sp, Prorrocaecum sp, Eimeria sp, Trichomonas sp, Serratospiculum sp, Isospora sp, e Microtetraneres $s p$. A análise parasitológica realizada nas amostras fecais mostrou-se um dispositivo adequado para auxiliar no controle das verminoses. Não obstante à prevalência de parasitos que foram detectadas em alta quantidade, os criadores não evidenciaram e nem relataram problemas sanitários nas aves. O trabalho concluiu que existe uma alta carga parasitária nas galinhas vendidas por essa propriedade, levando um sério risco para os humanos consumidores destas aves, como possíveis intoxicações alimentares.

Palavras-chave: Galinha caipira; Agricultura familiar; Feira livre; Parasitoses intestinais.

\begin{abstract}
s
This study aimed to establish the prevalence of parasitosis in domesticated hens, reared in extensive regime in the city of Porto Velho, Rondônia, Brazil. Forty bird fecal samples were analyzed in 4 (four) farms, 10 in each of them. The samples were then processed by the qualitative technique of Willis \& Mollay (simple flotation, 1921). Of the 40 stool samples analyzed, 32 were positive and 8 negative, and of these 32, 9 had mixed infections. The eggs of the parasites that were found in the exams are the following: Heterakis $s p$, Capillaria $s p$, Prorrocaecum $s p$, Eimeria $s p$,
\end{abstract}


Trichomonas sp, Serratospiculum sp, Isospora sp, and Microtetraneres sp. The parasitological analysis performed on fecal samples proved to be an adequate device to help control worms. Despite the prevalence of parasites that were detected in high quantities, the breeders did not show or report health problems in the birds. The work concluded that there is a high parasite load in the chickens sold by this property, leading to a serious risk for human consumers of these birds, such as possible food poisoning.

Keywords: Free-range chicken; Family farming; Free fair; Intestinal parasites.

\section{Resumen}

Este estudio tuvo como objetivo establecer la prevalencia de parasitosis en gallinas domesticadas, criadas en régimen extensivo en la ciudad de Porto Velho, Rondônia, Brasil. Se analizaron cuarenta muestras de heces de aves en 4 (cuatro) granjas, 10 en cada una de ellas. Las muestras fueron luego procesadas por la técnica cualitativa de Willis \& Mollay (flotación simple, 1921). De las 40 muestras de heces analizadas, 32 fueron positivas y 8 negativas, y de estas 32, 9 tenían infecciones mixtas. Los huevos de los parásitos que se encontraron en los exámenes son los siguientes: Heterakis sp, Capillaria sp, Prorrocaecum sp, Eimeria sp, Trichomonas sp, Serratospiculum sp, Isospora sp y Microtetraneres $s p$. El análisis parasitológico realizado en muestras fecales resultó ser un dispositivo adecuado para ayudar a controlar las lombrices. A pesar de la prevalencia de parásitos que se detectaron en grandes cantidades, los criadores no mostraron ni reportaron problemas de salud en las aves. El trabajo concluyó que existe una alta carga parasitaria en los pollos vendidos por esta propiedad, lo que genera un riesgo grave para los consumidores humanos de estas aves, como una posible intoxicación alimentaria.

Palabras clave: Gallina de granja; Agricultura familiar; Feria libre; Parásitos intestinales.

\section{Introdução}

A criação de aves no Brasil pode ser classificada como avicultura industrial e alternativa, sendo que a primeira é um dos setores agropecuários crescente no país, se destacando no mercado internacional devido à alta produtividade, qualidade e um menor custo de produção, enquanto o da avicultura alternativa, também chamada de semi-intensivo ou extensivo de produção, obteve destaque nos últimos anos (Alcântara, 2015; Mattos, Rossato \& Antonucci, 2019).

A criação extensiva de galinhas domesticadas, criadas em regime extensivo geralmente se faz por pequenos produtores, como fonte de renda alternativa, e por falta de recursos para grandes empreedimentos (Pinto, 2020; Sagrilo, 2007). A falta de fundos financeiros junto a incompreensão do produtor quanto a importância das helmintoses levam ao aumento da frequência de enfermidades nos modelos de criação extensivista (Cardozo \& Yamamura, 2004; Baboolal et al. 2012; Gomes et al. 2009; Siqueira \& Marques, 2016; Sobral et al. 2010) e semi-intensiva (Santana Neto et al. 2020).

Dentre as problemáticas na criação de aves de produção, destacam-se as parasitoses intestinais, associadas, geralmente, ao manejo não adequado dos produtores nas instalações, como a má higienização dos viveiros, a introdução de animais sem a devida quarentena, facilitando a infestação parasitária dos animais (Lima, 2011; Siqueira \& Marques, 2016), entre elas, as endoparasitoses (Rennó et al. 2008). Isto pode acarretar na perca econômica de renda extra, baixa produtividade e a altos índices de mortalidades em aves filhotes e jovens (Carneiro, 2001; Menezes, 1999).

Este tipo de criação de aves não possui instalações ou práticas de manejo que supra com eficiência as necessidades nutricionais, reprodutivas e sanitárias para esses animais (Quadros et al. 2015), sendo que as más condições podem vir a ser um obstáculo ao sucesso da atividade, devido ser uma fonte em potencial de proliferação de agentes patogênicos, tanto pela convivência de aves de várias espécies no mesmo ambiente quanto com humanos (Brandão et al. 2008; Fanatico, 2008). Com isso pode-se observar que os sistemas alternativos de produção de aves possuem um alto risco de infecções destas por patógenos, por possuírem um fácil acesso aos transmissores de possíveis parasitos, como aves selvagens, insetos, répteis e mamíferos (Melendez et al., 2010; Quadros et al. 2015). O trabalho teve como objetivo identificar os parasitos intestinais prevalentes em galinhas domésticas abatidas e vendidas nas feiras municipais na Cidade de Porto Velho, RO, Brasil.

O principal problema com a infestação parasitária em aves é a presença de uma imunossupressão, o que acarreta a possíveis agentes secundários, como as bactérias, vírus, micoplasma e fungos (Back, 2002). Os sinais clínicos que são 
observados em animais com endoparasitas são diarréias, abdômen dilatado, apatia, prostação, perda do apetite, perda de peso, má digestão dos alimentos e anemias (Back, 2002; Vasconcelos, 2000).

\section{Material e Métodos}

As amostras foram coletadas de 4 produtores em diferentes feiras municipais, localizadas na Cidade de Porto Velho, no estado de Rondônia (Brasil), durante os trinta dias do mês de Junho, do ano de 2019. Foram coletadas 40 amostras de vísceras de galinhas domésticas comercializadas por criadores rurais locais em feiras livres. Todo material foi acondicionado em isopor 450x25x402 mm contendo gelo e encaminhado e processado no Laboratório de Parasitologia de uma instituição de ensino superior. Foram coletadas amostras fecais, de aproximadamente $3 \mathrm{~g}$, sendo então realizada a técnica quantitativa de Willis \& Mollay (1921).

As 40 amostras de vísceras passaram por inspeção visual, onde foi realizado a incisão com o bisturi nos órgãos, principalmente os intestinos, com o intuito de averiguar se havia a presença de endoparasitas em seu estágio adulto que fossem visíveis a olho nu. De todas as amostras avaliadas, não houve a constatação da presença de parasitos adultos alojados nos órgãos dos animais.

Com isso, foi realizado o processamento das amostras fecais por meio da técnica de Willis \& Mollay do ano de 1921, afim de procurar possíveis ovos ou larvas de endoparasitos e diferenciá-los conforme o seu gênero. A técnica consiste em misturar em um Becker $1 \mathrm{~g}$ de fezes para 10ml de solução saturada, após isso utiliza-se uma gaze com uma peneira para filtrar o conteúdo para um Becker limpo. Depois desse processo, deve-se colocar o liquido filtrado em um recipiente de bordo perfeito, até o líquido formar um menisco no bordo do recipiente. Após isso, se coloca uma lâmina por cima do recipiente, com ela obtendo contato com o menisco. Então, após todo esse procedimento, deve-se deixar em repouso de um à dois minutos. Passado esse tempo, retira-se a lâmina com todo o cuidado. Com um movimento uniforme, retira-se a lamínula e a coloca sobre a lâmina, então leva-se a lâmina devidamente pronta para o microscópio e se inicia a busca por ovos de possíveis parasitos.

\section{Resultado e Discussões}

Das 40 amostras de fezes analisadas 32/40(80\%) foram positivas para a presença de endoparasitas e dentre elas 9/32 $(28,12 \%)$ apresentaram infecção mista. Foram identificados a presença de endoparasitos dos seguintes gêneros: Capillaria $s p$. 45,71\%, Heterakis sp. 24,76\%, Eimeria sp. 16,19\%, Prorrocaecum sp. 6,19\%, Trichomonas sp. 2,38\%, Serratospiculum sp. 1,42\%, Isospora sp. 0,95\% e Microtetraneres sp. 0,95\%, assim como apresentado na Tabela 1. 
Tabela 1 - Gêneros de endoparasitos encontrados nas 32 fezes e suas respectivas porcentagens.

\begin{tabular}{lc}
\hline Gênero & $\begin{array}{c}\text { Porcentagem } \\
(\%)\end{array}$ \\
Capillaria sp. & 45,71 \\
Heterakis sp. & 24,76 \\
Eimeria sp. & 16,19 \\
Prorrocaecum sp. & 6,19 \\
Trichomonas sp. & 2,38 \\
Serratospiculum sp. & 1,42 \\
Isospora sp. & 0,95 \\
Microtetraneres sp. & 0,95 \\
\hline Total & 98,55 \\
\hline
\end{tabular}

Fonte: Autores.

Costa e Freitas (1959) e Gomes et al. (2009) afirmam que no Brasil já foram identificadas 48 diferentes espécies de helmintos em galinhas domésticas. Neste presente trabalho, identificaram-se seis gêneros de helmintos e dois de coccídeo.

Segundo Sobral et al., (2010) a prevalência de endoparasitoses no plantel avícola brasileiro é próxima de 100\%, devido ao desconhecimento pela maior parte de criadores de aves, da necessidade de everminação dos animais criados em regime extensivo, nessa pesquisa observou-se uma positividade para a presença desses agentes em $80 \%$ das aves, número menor do que o já encontrado.

Siqueira et al., (2016) encontrou em sua pesquisa 92\% de prevalência das endoparasitoses em aves, valor próximo ao encontrado por Sobral et al. (2010). Dentre os agentes por eles encontrados, os endoparasitas do gênero Capillaria sp representam cerca de $84 \%$ dessa prevalência. Nesta pesquisa, a prevalência deste agente foi de $45,71 \%$, demonstrando-se inferior a prevalência encontrada pelos autores acima citados. No entanto, os endoparasitas do gênero Capillaria sp demostraram-se os de maior abundância nas amostras deste estudo.

Os cestóides são facilmente encontrados no regime de criação extensiva, pois requisitam a presença de hospedeiros intermediários (moluscos gastrópodes, minhocas, moscas, formigas ou outros insetos coprófagos) enquanto Eimeria sp., Capillaria sp., Heterakis gallinarum e Ascaridia galli tem ciclo direto. H. gallinarum tem importância por trasnportar um protozoário denominado Histomonas meleagridis, agente da histomonose ou "cabeça preta" das galinhas (Cardozo \& Yamamura, 2004). Contudo, os animais do estudo não apresentaram infestação por cestóides. Como a presença de vetores é necessário, pode-se levantar a hipótese de que, com esses vetores intermediários, os produtores têm tomado muito cuidado no meio produtivo.

Embury (1998) afirma que as helmintoses são as principais causas de enfermidades que acometem aves de criação, contribuindo para aumento da taxa de mortalidade, diminuição do ganho de peso e postura. No presente trabalho não foi possível constatar esses fatos, visto que foi feito apenas a averiguação da presença de helmintoses e outros endoparasitos nas aves comercializadas. Contudo, pode-se afirmar que a infestação parasitária em aves criadas em regime extensivo e comercializadas nas feiras da capital é ampla, visto que houve uma taxa de $80 \%$ das amostras acometidas por um ou mais endoparasito. 


\section{Conclusão}

Conclui-se que as aves vendidas em feira municipal na cidade de Porto-Velho apresentam uma elevada carga parasitaria, sendo possível idenficar agentes dos gêneros Capillaria sp., Heterakis sp. Eimeria sp., Prorrocaecum sp., Trichomonas sp., Serratospiculum sp., Isospora sp e Microtetraneres sp., demonstrando um risco para a população humana consumidora dessas aves vendidas em feiras da capital de Rondônia, como possíveis intoxicações alimentares.

\section{Referências}

Alcântara, J. B. (2015). Pesquisa de salmonella sp. em aves criadas em sistemaindustriale alternativo. Universidade federal de Goiás. Escola de veterinária e zootecnia. Programa de pós-graduação em ciência animal. Goiânia.

Baboolal, V. et al. (2012). The prevalence of intestinal helminths in broiler chickens in Trinidad. Veterinarski Arhiv, 82(6): 591-597.

Back, A. (2002). Manual de Doenças de Aves. BACK. 2002.

Brandão, P. A. et al. (2008). Prevalência de endoparasitoses em galinha caipira em assentamento rural no semi-árido paraibano. In: V Congresso Nordestino de Produção Animal, Aracaju, SE. Anais. SNPA/CNPA.

Caires, C. M., Carvalho, A. P. \& Caires, R. M. (2010). Criação Alternativa de Frangos de Corte. Nutritime.

Cardozo, S. P., \& Yamamura, M. H. (2004). Parasitas em produção de frangos no sistema de criação tipo colonial/caipira no Brasil. Semina Ciências Agrarias, $25(1), 63-74$.

Carneiro, V. S. (2001). Composição e estrutura da comunida-de de helmintos parasitos de galinhas, Gallus domesticus (L.), no Município de Seropédica, Estado do Rio de Janeiro. 2001. 69f. Dissertação (Mestrado em Parasitologia Veterinária) - Universidade Federal Rural do Rio de Janeiro.

Costa, H. M. A. \& Freitas, M. G. (1959). Novos achados helmintológicos em animais domésticos de Minas Gerais. Arquivos da Escola de Veterinária da UFMG, 12: 293-297.

Embrapa Meio-Norte. (2003). Validação do sistema alternativo de criação de galinha caipira. Sistemas de Produção. Versão eletrônica. Teresina: Agricultura familiar. 2003.

Fanatico, A. C. (2008). Performance, livability, and carcass yield of slow- and fast-growing Chicken genotypes fed low-nutrient or standard diets and raised indoors or with outdoor access. Poultry Science. 87(3):1012-21.

Embury, I. (2019). Raising guinea fowl.

Foley, S. L. et al. (2011). Population dynamics of Salmonella enterica serotypes in commercial egg and poultry production. Appl Environ Microbiol. 2011.

Gomes, F. F. et al. (2009). Principais parasitos intestinais diagnosticados em galinhas domésticas criadas em regime extensivo na municipalidade de Campos dos Goytacazes, RJ. Ciência Animal Brasileira, 10(3): 818-822.

Lima, E. M. et al. (2011). Perfil parasitológico intestinal de frangos caipiras criados em diferentes sistemas de criação. Anais 9o Seminário Anual de Iniciação Científica, Parapuapebas, AM, (Resumo 72).

Mattos, P. M., Rossato, M. R. \& Antonucci, A. M. (2019). Principais parasitos em aves industriais (frangos, galinhas e perus) - Revisão de Literatura. Revista científica de Medicina Veterinária, ISSN 1679-7353 Ano XVI - Número 32 - JANEIRO de 2019 - Periódico Semestral.

Melendez, S. N. et al. (2010). Salmonella entericaisolates from pasture-raised poultry exhibit antimicrobial resistance and class I integrons. J Appl Microbiol. Menezes, R. C. (1999). Helmintoses de galinhas d'angola (Nu-mida meleagris Linnaeus, 1758) criadas extensivamente no Estado do Rio de Janeiro, Brasil. 1999. 106 f. Disser-tação (Mestrado em Patologia Veterinária) - Universidade Federal Fluminense, Rio de Janeiro.

Pinto, I. M. (2020). Importância Da Assistência Técnica Na Criação De Galinhas Caipiras Para Corte. 27f. Dissertação para conclusão de curso para obtenção do grau de Zootecnista. Universidade católica de Goiás, Escola de Ciências Agrárias e biológicas Curso de Zootecnia, Goiânia, Goiás.

Quadros, R. M. et al. (2015). Prevalência de endo e ectoparasitos de galinhas caipiras em pequenas propriedades da região serrana de Santa Catarina. PUBVET, 9(1): 1-5.

Rennó, P. P. et al. (2008). Endoparasitose em aves-revisão de literatura. Revista Científica Eletretrônica de Medicina Veterinária, 6(11): 1-6.

REZENDE, I. R. et al. (2016). Inquérito parasitológico em frangos e galinhas caipiras do município São Luís de Montes Belos - GO. III Congresso de Ensino, Pesquisa e Extensão da UEG. Inovação: Inclusão Social e Direitos. Pirenópolis - GO. 19 a 21 de outubro de 2016.

Sagrilo, E. et al. (2007). Criação de galinhas caipiras. Embrapa / Informação Tecnológica. Embrapa Meio-Norte, Embrapa Informação Tecnológica, 73p.

Santana Neto, B. O. et al. (2020). Parasitas gastrointestinais em uma criação semi-intensiva de galinhas caipiras, no município de Carmo do Paranaíba, MG. PUBVET, 14(8): 163 .

Siqueira, G. B. \& Marques, S. M. T. (2016). Parasitos intestinais em galinhas caipiras da região metropolitana de Porto Alegre, RS. PUBVET, 10(9): 690-695. 
Research, Society and Development, v. 10, n. 9, e35710918081, 2021

(CC BY 4.0) | ISSN 2525-3409 | DOI: http://dx.doi.org/10.33448/rsd-v10i9.18081

Sobral, F. E. S., Brandão, P. A. \& Athayde, A. C. R. (2010). Utilização de fitoterápicos no tratamento de parasitoses em galinhas caipira criadas em sistema semi-extensivo. Agropecuária Científica do Semi-Árido, 6: 1- 6.

Vasconcelos, O. I. (2000). Parasitose em Aves de Produção Industrial. In: Junior, A.B., Macari, M. Doença das aves. FACTA.

Willis, H. H. (1921). A simple levitation method for the detection of wookworm ova. Medicine Journal of Australia, 8: 375-376. 